\title{
On Certain Properties of Trigonometrically $\rho$-Convex Functions
}

\author{
Mohamed Sabri Salem Ali \\ Department of Mathematics, Faculty of Basic Education, PAAET, Shamiya, Kuwait \\ Email: mss_ali5@yahoo.com
}

Received March 30, 2012; revised April 29, 2012; accepted May 11, 2012

\begin{abstract}
The aim of this paper is to prove that the average function of a trigonometrically $\rho$-convex function is trigonometrically $\rho$-convex. Furthermore, we show the existence of support curves implies the trigonometric $\rho$-convexity, and prove an extremum property of this function.
\end{abstract}

Keywords: Generalized Convex Functions; Trigonometrically $\rho$-Convex Functions; Supporting Functions; Average Functions; Extremum Problems

\section{Introduction}

In 1908, Phragmén and Lindelöf ( See, e.g. [1]) showed that if $F(z)$ is an entire function of order $0<\rho<\infty$, then its indicator which is defined as:

$$
h(\theta)=h_{F}(\theta)=\limsup _{r \rightarrow \infty} \frac{\log \left|F\left(r e^{i \theta}\right)\right|}{r^{\rho}},(\alpha \leq \theta \leq \beta)
$$

has the following property:

If $0<\rho(\beta-\alpha)<\pi$, and $H(\theta)$ is the function of the form

$$
H(\theta):=A \cos \rho \theta+B \sin \rho \theta
$$

(such functions are called sinusoidal or $\rho$-trigonometric) which coincides with $h(\theta)$ at $\alpha$ and at $\beta$, then for $\alpha \leq \theta \leq \beta$ we have

$$
h(\theta) \leq H(\theta) .
$$

This property is called a trigonometric $\rho$-convexity $([1,2])$.

In this article we shall be concerned with real finite functions defined on a finite or infinite interval $(a, b) \subset \mathbb{R}$.

A well known theorem [3] in the theory of ordinary convex functions states that: A necessary and sufficient condition in order that the function $f:(a, b) \rightarrow \mathbb{R}$, be convex is that there is at least one line of support for $f$ at each point $x$ in $(a, b)$.

In Theorem 3.1, we prove this result in case of trigonometrically $\rho$-convex functions. In Theorem 3.2, we prove the extremum property [4] of convex functions in case of trigonometrically $\rho$-convex functions. And finally in Theorem 3.3, we show that the average function [5] of a trigonometrically $\rho$-convex function is also trigonometrically $\rho$-convex.

\section{Definitions and Preliminary Results}

In this section we present the basic definitions and results which will be used later, see for example ([1,2], and [6-9]).

Definition 2.1. A function $f:(a, b) \rightarrow \mathbb{R}$ is said to be trigonometrically $\rho$-convex if for any arbitrary closed subinterval $[u, v]$ of $(a, b)$ such that

$0<\rho(v-u)<\pi$, the graph of $f(x)$ for $x \in[u, v]$ lies nowhere above the $\rho$-trigonometric function, determined by the equation

$$
H(x)=H(x ; u, v, f)=A \cos \rho x+B \sin \rho x
$$

where $A$ and $B$ are chosen such that $H(u)=f(u)$, and $H(v)=f(v)$.

Equivalently, if for all $x \in[u, v]$

$$
f(x) \leq H(x)=\frac{f(u) \sin \rho(v-x)+f(v) \sin \rho(x-u)}{\sin \rho(v-u)} .
$$

The trigonometrically $\rho$-convex functions possess a number of properties analogous to those of convex functions.

For example: If $f:(a, b) \rightarrow \mathbb{R}$ is trigonometrically $\rho$-convex function, then for any $u, v \in(a, b)$ such that $0<\rho(v-u)<\pi$, the inequality $f(x) \geq H(x ; u, v, f)$ holds outside the interval $(u, v)$.

Definition 2.2. A function 


$$
T_{u}(x)=A \cos \rho x+B \sin \rho x
$$

is said to be supporting function for $f(x)$ at the point $u \in(a, b)$, if

$$
T_{u}(u)=f(u) \text {, and } T_{u}(x) \leq f(x) \forall x \in(a, b) .
$$

That is, if $f(x)$ and $T_{u}(x)$ agree at $x=u$ and the graph of $f(x)$ does not lie under the support curve.

Remark 2.1. If $f:(a, b) \rightarrow \mathbb{R}$ is differentiable trigonometrically $\rho$-convex function, then the supporting function for $f(x)$ at the point $u \in(a, b)$ has the form

$$
T_{u}(x)=f(u) \cos \rho(x-u)+f^{\prime}(u) \sin \rho(x-u) .
$$

Proof. The supporting function $T_{u}(x)$ for $f(x)$ at the point $u \in(a, b)$ can be described as follows:

$$
T_{u}(x)=\lim _{v \rightarrow u} H(x ; u, v, f),
$$

where $v \in(a, b)$ such that $0<\rho(v-u)<\pi$, and as

$$
f(x) \geq H(x ; u, v, f), \forall x \in(a, b) \backslash(u, v) .
$$

Then taking the limit of both sides as $v \rightarrow u$, and from (1), one obtains

$$
\begin{aligned}
& f(x) \geq T_{u}(x) \\
& =\lim _{v \rightarrow u} H(x ; u, v, f) \\
& =\lim _{v \rightarrow u} \frac{f(u) \sin \rho(v-x)+f(v) \sin \rho(x-u)}{\sin \rho(v-u)} \\
& =f(u) \cos \rho(x-u)+f^{\prime}(u) \sin \rho(x-u) .
\end{aligned}
$$

Thus, the claim follows.

Theorem 2.1. A trigonometrically $\rho$-convex function $f:(a, b) \rightarrow \mathbb{R}$ has finite right and left derivatives $f_{+}^{\prime \prime}(x), f_{-}^{\prime}(x)$ at every point $x \in(a, b)$, and $f_{-}^{\prime}(x) \leq f_{+}^{\prime \prime}(x)$ for all $x \in(a, b)$.

Theorem 2.2. Let $f:(a, b) \rightarrow \mathbb{R}$ be a two times continuously differentiable function. Then $f$ is trigonome-trically $\rho$-convex on $(a, b)$ if and only if $f^{\prime \prime}(x)+\rho^{2} f(x) \geq 0$ for all $x \in(a, b)$.

Property 2.1. Under the assumptions of Theorem 2.1, the function $f$ is continuously differentiable on $(a, b)$ with the exception of an at most countable set.

Property 2.2. A necessary and sufficient condition for the function $f(x)$ to be a trigonometrically $\rho$-convex in $(a, b)$ is that the function

$$
\varphi(x)=f^{\prime}(x)+\rho^{2} \int_{w}^{x} f(t) \mathrm{d} t, \quad w \in(a, b)
$$

is non-decreasing in $(a, b)$.

Lemma 2.1. Let $f:(a, b) \rightarrow \mathbb{R}$ be a continuous, $2 \pi$ periodic function, and the derivative $f^{\prime}(x)$ exists and piecewise continuous function and let $M$ be a set of discontinuity points for $f^{\prime}(x)$ If

$$
f^{\prime}\left(x_{k}-0\right) \leq f^{\prime}\left(x_{k}+0\right), x_{k} \in M,
$$

and $f \in C^{2}((a, b) \backslash M)$, where

$$
f^{\prime \prime}(x)+\rho^{2} f(x) \geq 0, \quad x \in(a, b) \backslash M .
$$

Then $f(x)$ is trigonometrically $\rho$-convex on $(a, b)$. Proof. Consider

$$
\varphi(x)=f^{\prime}(x)+\rho^{2} \int_{w}^{x} f(t) \mathrm{d} t, w \in(a, b) .
$$

Two cases arise, as follows.

Case 1. Suppose $x=x_{k} \in M$. Using (5), we observe

$$
\varphi\left(x_{k}+0\right)-\varphi\left(x_{k}-0\right)=f^{\prime}\left(x_{k}+0\right)-f^{\prime}\left(x_{k}-0\right) .
$$

From (3), we get $\varphi\left(x_{k}+0\right) \geq \varphi\left(x_{k}-0\right)$.

So, the function $\varphi(x)$ is non-decreasing in $M$. Case 2. Let $x \in\left(x_{k-1}, x_{k}\right), x_{k-1}, x_{k} \in M$, and $\left(x_{k-1}, x_{k}\right) \cap M=\varnothing$.

Differentiating both sides of (5) with respect to $x$, one has

$$
\varphi^{\prime}(x)=f^{\prime \prime}(x)+\rho^{2} f(x) .
$$

Using (4), one obtains

$$
\varphi^{\prime}(x) \geq 0, x \in\left(x_{k-1}, x_{k}\right) .
$$

Thus, $\varphi(x)$ is non-decreasing function in $\left(x_{k-1}, x_{k}\right)$.

Therefore, from Property 2.2, we conclude that the function $f(x)$ is trigonometrically $\rho$-convex on $(a, b)$.

\section{Main Results}

Theorem 3.1. A function $f:(a, b) \rightarrow \mathbb{R}$ is trigonometrically $\rho$-convex on $(a, b)$ if and only if there exists a supporting function for $f(x)$ at each point $x$ in $(a, b)$.

Proof. The necessity is an immediate consequence of F. F. Bonsall [10].

To prove the sufficiency, let $x$ be an arbitrary point in $(a, b)$ and $f$ has a supporting function at this point. For convenience, we shall write the supporting function in the follwoing form:

$$
T_{x}(z)=f(x) \cos \rho(z-x)+K_{x, f} \sin \rho(z-x),
$$

where $K_{x, f}$ is a fixed real number depends on $x$ and $f$.

From Definition 2.2, one has

$$
T_{x}(x)=f(x) \text {, and } T_{x}(z) \leq f(z) \forall z \in(a, b) .
$$

It follows that,

$$
f(x) \cos \rho(z-x)+K_{x, f} \sin \rho(z-x) \leq(z) \forall z \in(a, b) .
$$

For all $u, v \in(a, b)$, choose any $u \neq v$ such that $0<\rho(v-u)<\pi$, and $\lambda, \mu \geq 0$ with $\lambda+\mu=1$ and let 
$x=\lambda u+\mu v$.

Applying (6) twice at $z=u$ and at $z=v$ yields

$$
\begin{aligned}
& f(x) \cos \rho(u-x)+K_{x, f} \sin \rho(u-x) \leq f(u), \\
& f(x) \cos \rho(v-x)+K_{x, f} \sin \rho(v-x) \leq f(v) .
\end{aligned}
$$

Multiplying the first inequality by $\sin \rho \lambda(v-u)$, the second by $\sin \rho \mu(v-u)$, and adding them, we obtain

$$
\begin{aligned}
& f(x)[\sin \rho \lambda(v-u) \cos \rho(u-x) \\
& -\cos \rho(v-x) \sin \rho \mu(u-v)] \\
& +K_{x, f} \sin \rho \lambda(v-u) \sin \rho(u-x) \\
& -\sin \rho(v-x) \sin \rho \mu(u-v) \\
& \leq f(u) \sin \rho \lambda(v-u)+f(v) \sin \rho \mu(v-u) .
\end{aligned}
$$

Consequently

$$
f(x) \leq \frac{f(u) \sin \rho(v-x)+f(v) \sin \rho(x-u)}{\sin \rho(v-u)}
$$

for all $x \in[u, v]$, which proves that the function $f(x)$ is trigonometrically $\rho$-convex on $(a, b)$.

Hence, the theorem follows.

Remark 3.1. For a trigonometrically $\rho$-convex function $f:(a, b) \rightarrow \mathbb{R}$, the constant $K_{x, f}$ in the above theorem is equal to $f^{\prime}(x)$ if $f$ is differentiable at the point $x$ in $(a, b)$, otherwise, $K_{x, f} \in\left[f_{-}^{\prime}(x), f_{+}^{\prime \prime}(x)\right]$.

Theorem 3.2. Let $f:(a, b) \rightarrow \mathbb{R}$ be a trigonometrically $\rho$-convex function such that $0<\rho(b-a)<\pi$ and let $T_{u}(x)$ be a supporting function for $f(x)$ at the point $u \in(a, b)$. Then the function

$$
G(u)=\int_{a}^{b}\left[f(x)-T_{u}(x)\right] \mathrm{d} x
$$

has a minimum value at $u=\frac{a+b}{2}$.

Proof. From Definition 2.2, we have

$$
T_{u}(u)=f(u),
$$

and

$$
T_{u}(x) \leq f(x) \quad \forall x \in(a, b),
$$

and $T_{u}(x)$ can be written in the form

$$
\begin{aligned}
T_{u}(x) & =f(u) \cos \rho(x-u)+K_{u, f} \sin \rho(x-u) \\
& =K \sin \rho(x+\alpha-u),
\end{aligned}
$$

where $K=\sqrt{f^{2}(u)+K_{u, f}^{2}}$, and $\tan \rho \alpha=\frac{f(u)}{K_{u, f}}$.

Using (9), one obtains

$$
\begin{aligned}
& \int_{a}^{b} T_{u}(x) \mathrm{d} x \\
& =K \int_{a}^{b} \sin \rho(x+\alpha-u) \mathrm{d} x \\
& =\frac{2}{\rho} \sin \rho\left(\frac{b-a}{2}\right) K \sin \rho\left[\left(\frac{b+a}{2}\right)+\alpha-u\right] \\
& =\frac{2}{\rho} \sin \rho\left(\frac{b-a}{2}\right) T_{u}\left(\frac{a+b}{2}\right)
\end{aligned}
$$

Consequently,

$$
G(u)=\int_{a}^{b} f(x) \mathrm{d} x-\frac{2}{\rho} \sin \rho\left(\frac{b-a}{2}\right) T_{u}\left(\frac{a+b}{2}\right) .
$$

Using (7) at $u=\frac{a+b}{2}$, the function $G(u)$ becomes

$G\left(\frac{a+b}{2}\right)=\int_{a}^{b} f(x) \mathrm{d} x-\frac{2}{\rho} \sin \rho\left(\frac{b-a}{2}\right) f\left(\frac{a+b}{2}\right)$.

But from (8), we observe $T_{u}\left(\frac{a+b}{2}\right) \leq f\left(\frac{a+b}{2}\right)$ for all $u \in(a, b)$.

Now using (10) and (11), it follows that $G(u) \geq G\left(\frac{a+b}{2}\right)$ for all $u \in(a, b)$.

Hence, the minimum value of the function $G(u)$ occurs at $u=\frac{a+b}{2}$.

Theorem 3.3. Let $f(x)$ be a non-negative, $2 \pi$ periodic, and trigonometrically $\rho$-convex function with a continuous second derivative on $\mathbb{R}^{+}$and let $F(x)$ be a $2 \pi$-periodic function defined in $[0,2 \pi]$ as follows

$$
F(x)=\frac{1}{x} \int_{0}^{x} f(t) \mathrm{d} t, \quad x \in[0,2 \pi] .
$$

If $f^{\prime}(0) \geq 0$, and

$$
f(0)=\frac{1}{2 \pi} \int_{0}^{2 \pi} f(t) \mathrm{d} t
$$

Then, $F(x)$ is trigonometrically $\rho$-convex function.

Proof. The proof mainly depends on Lemma 2.1. So, we show that the function $F(x)$ satisfies all conditions in this lemma.

Suppose that

$$
g(x):=\frac{1}{x} \int_{0}^{x} f(t) \mathrm{d} t, x \in \mathbb{R}^{+} .
$$

It is obvious that, $g(0)=f(0)$.

First, we study the behavior of the function $F(x)$ inside the interval $(0,2 \pi)$.

It is clear from (12) that $F(x) \mathrm{s}$ is an absolutely 
continuous function, has a derivative of third order.

But from the periodicity of $F(x)$ and (13), we get

$$
\begin{aligned}
F(0) & =g(0)=f(0) \\
& =\frac{1}{2 \pi} \int_{0}^{2 \pi} f(t) \mathrm{d} t=g(2 \pi)=F(2 \pi) .
\end{aligned}
$$

Using the following substitution $t=x \tau$.

It follows that, $F(x)$ can be written as

$$
F(x)=\int_{0}^{1} f(x \tau) \mathrm{d} \tau \text { and } F^{\prime \prime}(x)=\int_{0}^{1} \tau^{2} f^{\prime \prime}(x \tau) \mathrm{d} \tau \text {. }
$$

Consequently,

$$
\begin{aligned}
& F^{\prime \prime}(x)+\rho^{2} F(x) \\
& =\int_{0}^{1} \tau^{2}\left(f^{\prime \prime}(x \tau)+\rho^{2} f(x \tau)\right)+\rho^{2}\left(1-\tau^{2}\right) f(x \tau) \mathrm{d} \tau .
\end{aligned}
$$

Since $f(x)$ is non-negative, trigonometrically $\rho$-convex function, and $0 \leq \tau \leq 1$, then from Theorem 2.2 and (16) it follows that

$$
F^{\prime \prime}(x)+\rho^{2} F(x) \geq 0, x \in(0,2 \pi) .
$$

Second, we prove that

$$
F^{\prime}(2 \pi-0) \leq F^{\prime}(2 \pi+0) .
$$

From the definition of $g(x)$ in (14) and the periodicity of $F(x)$, we observe that

$F^{\prime}(2 \pi-0)=g^{\prime}(2 \pi)$, and $F^{\prime}(2 \pi+0)=g^{\prime}(0)$.

Again using (14), we have

$$
g^{\prime}(x)=\frac{f(x)-g(x)}{x} .
$$

Thus, from (15) and (19), one has $g^{\prime}(2 \pi)=0$, and $g^{\prime}(0)=\frac{1}{2} f^{\prime}(0)$.

Hence, from (13), we infer that

$$
\begin{aligned}
F^{\prime}(2 \pi-0) & =g^{\prime}(2 \pi)=0 \leq \frac{1}{2} f^{\prime \prime}(0) \\
& =g^{\prime}(0)=F^{\prime}(2 \pi+0),
\end{aligned}
$$

and the inequality in (18) is proved.

Now using (17), (18), and Lemma 2.1, we conclude that $F(x)$ is trigonometrically $\rho$-convex function, and the theorem is proved.

\section{Acknowledgements}

The author wishes to thank the anonymous referees for their fruitful comments and suggestions which improved the original manuscript.

\section{REFERENCES}

[1] B. Ya. Levin, "Lectures on Entire Functions," American Mathematical Society, 1996.

[2] L. S. Maergoiz, "Asymptotic Characteristics of Entire Functions and Their Applications in Mathematics and Biophysics," Kluwer Academic Publishers, New York, 2003.

[3] A. W. Roberts and D. E. Varberg, "Convex Functions," Academic Press, New York, 1973.

[4] M. J. Miles, "An Extremum Property of Convex Functions," American Mathematical Monthly, Vol. 76, 1969, pp. 921-922. doi:10.2307/2317948

[5] A. M. Bruckner and E. Ostrow, "Some Functions Classes Related to the Class of Convex Functions," Pacific Journal of Mathematics, Vol. 12, 1962, pp. 1203-1215.

[6] E. F. Beckenbach, "Convex Functions," Bulletin of the American Mathematical Society, Vol. 54, No. 5, 1948, pp. 439-460. doi:10.1090/S0002-9904-1948-08994-7

[7] J. W. Green, "Support, Convergence, and Differentiability Properties of Generalized Convex Functions," Proceedings of the American Mathematical Society, Vol. 4, No. 3, 1953, pp. 391-396. doi:10.1090/S0002-9939-1953-0056039-2

[8] M. M. Peixoto, "On the Existence of Derivatives of Generalized Convex Functions," Summa Brasilian Mathematics, Vol. 2, No. 3, 1948, pp. 35-42.

[9] M. M. Peixoto, "Generalized Convex Functions and Second Order Differential Inequlities," Bulletin of the American Mathematical Society, Vol. 55, No. 6, 1949, pp. 563572. doi:10.1090/S0002-9904-1949-09246-7

[10] F. F. Bonsall, "The Characterization of Generalized Convex Functions," The Quarterly Journal of Mathematics Oxford Series, Vol. 1, 1950, pp. 100-111. doi:10.1093/qmath/1.1.100 